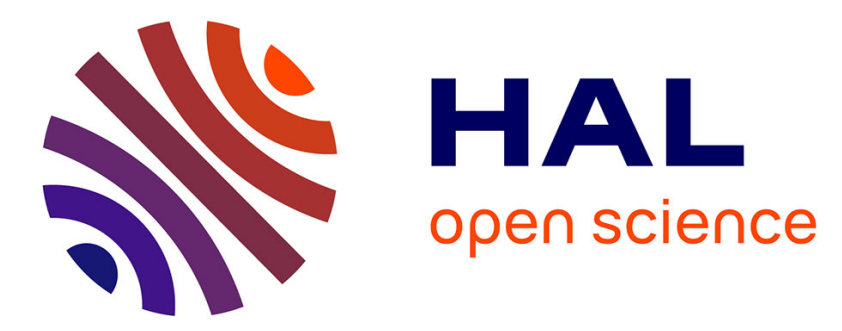

\title{
SSR mining in coffee tree EST databases: potential use of EST-SSRs as markers for the Coffea genus
}

\author{
Valérie Poncet, Myriam Rondeau, Christine Tranchant, Anne Cayrel, Serge
}

Hamon, Alexandre De Kochko, Perla Hamon

\section{To cite this version:}

Valérie Poncet, Myriam Rondeau, Christine Tranchant, Anne Cayrel, Serge Hamon, et al.. SSR mining in coffee tree EST databases: potential use of EST-SSRs as markers for the Coffea genus. Molecular Genetics and Genomics, 2006, 276 (5), pp.436-449. 10.1007/s00438-006-0153-5 . ird-01223821

\section{HAL Id: ird-01223821 \\ https://hal.ird.fr/ird-01223821}

Submitted on 6 Nov 2015

HAL is a multi-disciplinary open access archive for the deposit and dissemination of scientific research documents, whether they are published or not. The documents may come from teaching and research institutions in France or abroad, or from public or private research centers.
L'archive ouverte pluridisciplinaire HAL, est destinée au dépôt et à la diffusion de documents scientifiques de niveau recherche, publiés ou non, émanant des établissements d'enseignement et de recherche français ou étrangers, des laboratoires publics ou privés. 


\title{
SSRminingincoffeetreeESTdatabases: potentialuseofEST-SSRsasmarkersfortheCoffeagenus
}

\author{
ValériePoncet $\cdot$ MyriamRondeau $\cdot$ \\ ChristineTranchant·AnneCayrel-SergeHamon·AlexandredeK \\ ochko·PerlaHamon
}

\begin{abstract}
Expressedsequencetags(ESTs)fromCoffea canephoraleavesandfruitswereusedtosearchfortypesan dfrequenciesofsimplesequencerepeats(ESTSSRs)withamotiflengthof 1-6bp.Fromanonredundant(NR)ESTsetof5,534potentialunigenes, $6.8 \%$ SSRcontainingsequenceswereidentified, withanaverageden sityofoneSSRevery7.73kbofESTsequences.Trinucleoti derepeatswerefoundtobethemostabundant(34.34\%),fol lowedbydi-(25.75\%)andhexanucleotide(22.04\%)motifs. Thedevelopmentofuniqueg enicSSRmarkerswasopti-

mizedbyacomputationalapproachwhichallowedustoeli minateredundancyintheoriginalESTsetandalsototestthe specificityofeachpairofdesignedprim-ers. Twenty-

fiveEST-SSRsweredevelopedandusedtoevaluatecrossspeciestransferabilityintheCoffea

genus. Theorthologywassupportedbytheampliconseque ncesimilarityandtheamplificationpatterns. The

>94\%identityofflankingsequencesrevealedhighsequence conservationacrossthe Coffea

genus.Ahighlevelofpolymorphiclociwasobtainedregardle ssofthespeciesconsidered(from $75 \%$ for

C.libericato86\% forC.canephora).Moreover,thepolymorp hismrevealedbyEST-

SSR wassimilartothatexposedbygenomicSSR.Itisconclud edthat Coffea

ESTsareavaluableresourceformicrosatellitemining.ESTSSRmarkersdevelopedfromC.canephorasequencescan
\end{abstract}

\footnotetext{
V.Poncet(\&)·M.Rondeau.C.Tranchant·A.Cayrel.

S.Hamon·A.deKochko·P.Hamon

UMR1097DiversitéetGénomesdesPlantesCultivées( DGPC),IRD,InstitutdeRecherchepourleDéveloppeme nt,911 avenueAgropolis,BP64501,

34394MontpellierCedex 5,Francee-

mail:poncet@mpl.ird.fr

URL:http://www.dgpc.org/index.html
}

beeasilytransferredtootherCoffea

speciesforwhichverylittlemolecularinformationisavailabl e.Theyconstituteasetofconservedorthologousmarkers, whi chwouldbeidealforassessinggeneticdiversityincoffeetrees aswellasforcross-

referencingtranscribedsequencesincomparativegenomics studies.

Keywords SSRmining.EST-SSR.Coffea

-Transferability. Geneticvariation

\section{Introduction}

Microsatellitemarkersderivedfromanonymousgenomicsequenceshavebeenextensivelyusedoverthelastdec adebecauseoftheirhighlyinterestingproperties(Ellegren 2004). Thesegenerallyco-dominantandlocusspeciWcmarkershaverevealedhighpolymorphismlevels.Goodtransferabilitybetweenspeciesandso metimesbetweengenerahasoftenbeenreported(Dirlewa ngeretal.2002; Ralloetal.2003;Gonzalez-

Martinezetal.2004;Poncetetal.2004).Thesefeatureshav eproventobeofgreatinterestforgeneticdiversitystudies, $g$ eneticandcomparativemapping(WuandTanksley 1993; Gonzaloetal.2005;Sahaetal.2005;Vigourouxetal.2005). However,despitetheseadvan-

tages, theyoftencorrespondtonon-

codingsequencesandthuscannothelpinseekingcandidate genes.

Expressedsequencetags(ESTs)aresequencedportionsof complementaryDNAcopiesof mRNAtheyrepresentpartofthetranscribedportionofthegenome ingivenconditions.Asexpected, theymainlycorrespondtorelativelyconservedsequences. Techniqueshave beendevelopedtorevealpolymorphismassociatedwithsuchsequences(Catoetal.2001),andstudies

basedonintronpolymorphism(lessconservedgenicregions)h avealsobeencarriedout(LemandLalle-

mand2003).However,ESTsequenceanalysesrevealedappro 
ximately $1.5-7.5 \%$ ofsequencescon-

tainingmicrosatellitemotifsincereals(Kantetyetal.2002;Th ieletal.2003).Amongdicotyledonousspe-

cies, thefrequencyofESTscontainingSSRswasfound

torangefrom2.65to16.82\%(KumpatlaandMukho-

padhyay2005).Therefore, regardlessoftheplantcon-

sidered, anever-

increasingnumberofESTsequencesprovidesacomplement arysourceformicrosatellitemarkeridentification.Although theconservednatureofcodingsequencesmaylimittheirpoly morphism,itshould facilitatecrossamplificationoflociamongphy-

logeneticallyrelatedspecies(Scottetal.2000)andevengener a.Moreover,theyhaveahighprobabilityofbeingassociated withfunctionalportionsofthegenome.Amongtheirmanyap plications, theseEST-derivedmarkers(EST-

SSR)canbeusedtocross-ref-

erencegenesbetweenspeciesforenhancingtheresolutionincomparativegenomicsstudiesandidentifyingconserv edgenomicregionsamongspeciesandgenera(Brownetal.20 01;Decroocqetal.2003; Guptaetal.2003;Sahaetal.2004; Yu etal.2004;Parketal.2005; Varshneyetal.2005b).

\section{Inthe Coffea}

genus,SSRmarkershaverecentlybecomemoreavailable throughtheconstructionofenrichedgenomicmicrosatellit elibraries(Rovellietal.2000;Dufouretal.2001;Baruaheta 1.2003).Microsat-

ellitemarkerswerefurtherevaluatedforamplificationam ong Coffea species(Baruahetal.2003; MoncadaandMcCouch2004; Poncetetal.2004).Goodcross-

speciestransferabilityandhighgeneticdiversityweregene rallyobserved.However,onlyahandfuloflinkagemapsha vebeenconstructed,includingthesemarkers(Lashermese tal.2001;Coulibalyetal.2003; N'Diayeetal.2006). These mapsweremainlyconstructedusingAFLPmarkersbutthe irrelevanceforcoffeetreebreedingprogramsislimited.M oreover,currentCoffea

mapsderivedfromdifferentCoffea

specieshardlyeversharean adequate numberof common (anchor)mark-erstobeabletobridgemaps.

PublicaccessibilitytoCoffea ESTdatabasesislimitedtoanSSHlibraryof527non-

redundant(NR)ESTsequencesassociated with

reactionsto

the

rustfungus(Fernandezetal.2004).Theseresourceswerev eryrecentlyenhancedbythegenerationof 13,175 unigenesfromC.canephora(Linetal.2005).OnlynineESTSSR

markershavebeendevelopedtodate(Bhatetal.2005).

WerecentlydevelopedtwoC.canephoraESTsequenc esetsinourlaboratoryfromcDNAisolatedfromleavesand fruitsatdifferentdevelopmentand maturationstages(totalof 10,420sequences,unpublisheddata).Thefirstobjectiveofthepresentstudywastoiden tifyandcharacterizemicrosatellitespresentintheNRsetofou rESTstoevaluateitspotentialasasourceformarkerdevelop ment.Thesecondaimwastodevelopasetofhighlypolymorp hicmarkersthatcouldcross-

amplifyanddistinguishbetweenCoffea species.

HerewereportthedevelopmentofEST-SSRmarkersbasedonC.canephorasequencesandtheircross-

speciestransferabilitytosix Coffea

species.Locusorthologywasmonitoredbyanalyzingamplif icationpatternsandbysequencingsomeamplicons.Relevan tinformationaboutthemutationmodelandtheevolu-

tionoftheselociwasalsonoted.Polymorphismsdetectedwit hinandbetweenasetofCoffea

specieswasalsoanalyzedtoassesswhetherthesemarkerscou ldbeusefulfordiversitystudiesanddistinguishingbetween $C$ offea

species. Weidentifiedasetofanchormarkers, mostofthemwi thingenesoffunctionalrelevance, whichwouldbehelpfulfor functionalandcomparativemappingwithinthe Coffea genus.

\section{Materialsandmethods}

PlantDNA

CoVee treesweremaintainedin a tropical greenhouseattheIRDresearchcenterinMontpellier

(France).

TotalDNAfromfullydevelopedleaveswasextracteda ccordingtoKyetal.(2000).Sevenspecies(C.canephora Pierre,knownasRobusta (CAN), C.eugenioides Moore(EUG),C.heterocalyxStoVelen(HET), C.libericaPortères(LIB),C.dewevre iPortère s(DEW),C.sp.Moloundou(MOL), and

C.pseudozanguebariae Bridson(PSE))representingthethr eeAfricanmaingeographicalclades(Lasher-

mesetal.1997)wereanalyzed.Polymorphismwasassessedi n12CAN,10DEW,10PSEand8LIB,rep-

resentativeofthegeneticdiversityofthe fourspecies,plus2EUG,2MOL, andtheonlyknownHETindividual

\section{DataminingforSSRmarkers}

ThecoffeeESTdatabasesusedinthisprojectweredevelopedi nourlaboratory(deKochkoetal.,unpub-

lisheddata).Theycontained 10,420sequencesderivedfromf ruit $(5,814$ sequences)andleaf $(4,606) \mathrm{cDNAlibraries}$ (valid sequencessubmittedtoGenBankunderaccessionnumbersE E191792-

EE200565).TherawchromatogramswereprocessedusingE STdbsoftware 
(http://www.mpl.ird.fr/bioinfo/).ESTdbisasetofanalyticalproceduresthatautomaticallyverify,cleanse,storeanda nalyzeESTsgeneratedinourlaboratory.ESTdbhasthreemajo rcomponents:(1)apipelineanal-

ysisprogrambasedonpublicsoftwareintegratedbyanin-

housedevelopedscript,(2)theresultsandrelatedinformationa restoredinarelationaldatabaseaccessi-

blethrough(3)awebinterface(Fig.1).ThefunctionsoftheEST sequenceswerepredictedthroughsimilaritysearchesfrompro teinsequenceGenBankdatabases(http://www.ncbi.nlm.nih. gov)usingBLASTx(Altschuletal.1997).Potentialunigenes( contigsandsingletonsfromallESTsequencesprocessedsimul taneously)wereidentifiedafter clustering for aNRESTset.

Theseunigenesequenceswerescreenedforthepresenceo fperfectandimperfectmicrosatellitesusingSSRpipeline,aP erlscriptdevelopedbyDubreuil-

Tranchant(SSRpipelineispubliclyavailableathttp://www. mpl.ird.fr/bioinfo/). Thisprogramintegratedthree publicsoftware packages:(1)a PerlprogramdevelopedbyCartingour(http://www.gramene.org)whichdetect sperfectmicrosatellites,(2)RepeatTandemFinder, anSSRrepeatWnder(http://www.tan-dem.bu.edu), and (3)Primer3,aPCRprimerdesignprogram(RozenandSkalet sky2000).Moreover,thisprogramallowedustocheckthespe cificityofprimerpairsbyblastingagainsttheESTsequences. ThistoolscreenedeachsequenceforSSRs. Theparametersw eresetfordetectionofmono-,di-,tri-,tetra-,penta-

,andhexanucleotidemotifswithaminimumof 15,9,6,5,4, an d3repeats,respectively.Themajorprimerdesignparameters weresetasfollows:primerlengthfrom 18to21bp(optimum2 0),PCRproductsizefrom 100to300bp,optimumannealingte mperature $60^{\circ} \mathrm{C}$.

Asetof25EST-

SSRprimerswasfurtheranalyzed(sequencessubmittedtoG enBankunderaccessionnumbersDQ778713-

DQ778737).Theywereselectedbasedonthefeatureoftheseq uence:wekeptinpriority
Fig.1 Flowchartof the clusteringandannotationofESTsvi $a$ theIRDpipelineESTdb, data miningofSSR-containingESTsandprimerdesignfora non-redundantSSRset

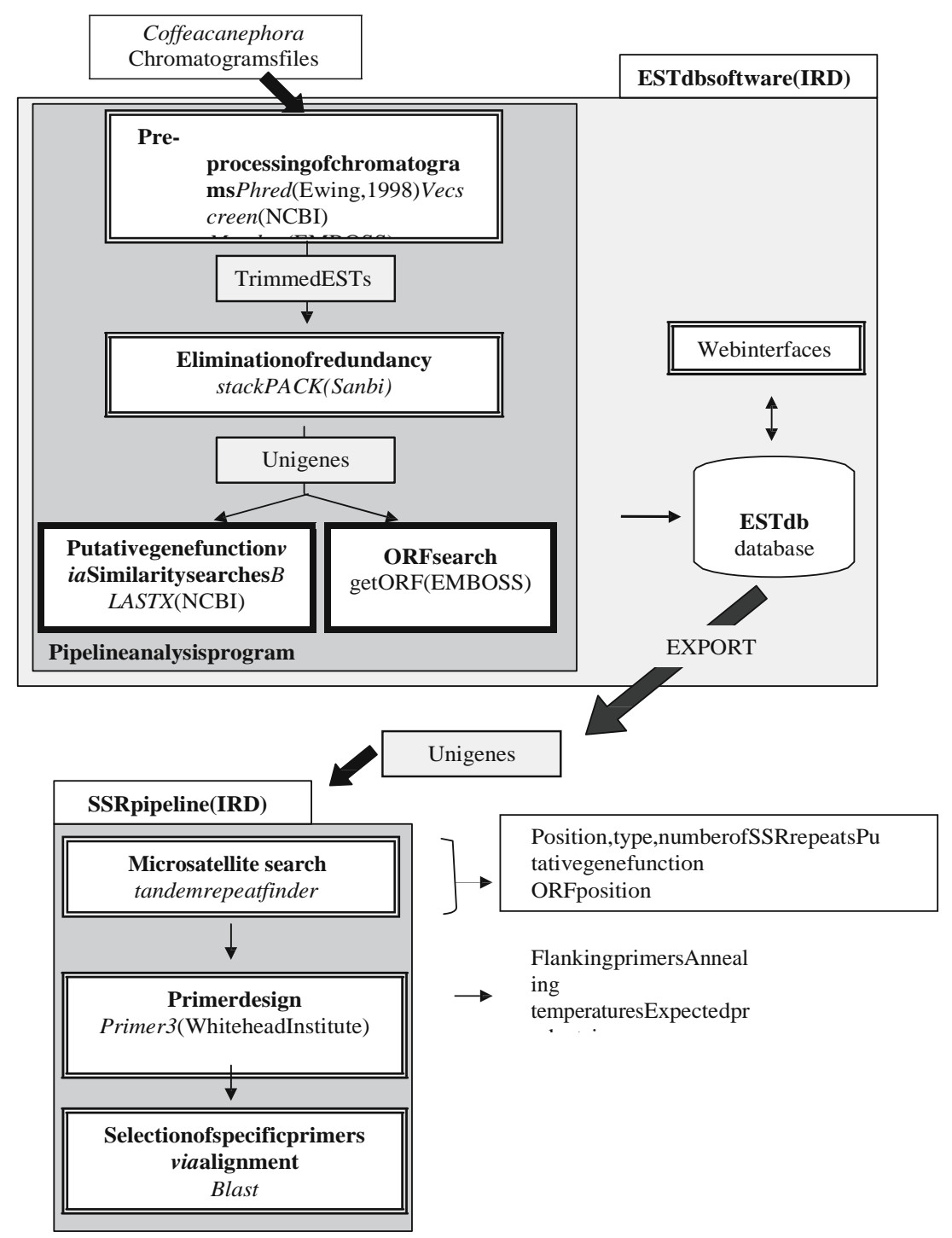


singletonESTs.Everyforwardprimerwas5-

tailedwiththeM13sequence5-

CACGACGTTGTAAAACGAC-

3. TheprimersweresynthesizedbyMWG-

BiotechAG(Ebersberg,Germany).

Polymerasechainreaction(PCR)amplificationand

visualisationofamplicons

Polymerasechainreactionamplificationswerecarriedout asdescribedinCoulibalyetal.(2003)andPoncetetal.(200

4)usingatouchdownPCRprofileoptimisedforeach

setofprimers: touchdown $60-55^{\circ} \mathrm{C}$ ortouch-down55-

$50^{\circ} \mathrm{C} . \mathrm{PCR}$ productsweredetectedonanIR ${ }^{2}$ AutomatedD

NASequencer(LI-COR,model4200L-

2,Lincoln,NE,USA)usinganM13primercoupledtothein fraredtagIRD700orIRD800andaftermigra-

tionon $25 \mathrm{~cm} 6.5 \% \mathrm{~KB}$ plus(LI-COR,CAT\#827-

05607)polyacrylamidegels.

ThegelimageswereprocessedbySAGAGT ${ }^{\mathrm{TM}_{\mathrm{M}}}$ software( LICORBiotech)toestimatethesizeofampliconsaccordingt oa50-350bpsizestandard(LI-COR,CAT\#829-05343,82905344).

Microsatellitecloningandsequencing

GenomicDNAswereamplifiedwiththeappropriateforw ardandreverseprimersbutwithouttheinfraredfluorescent M13.TheproductswerepurifiedusingEZNACyclePure(OMEGABio-

Tek,Doraville,GA,USA)andclonedontothepCRß4-

TopoplasmidusingtheTOPOTAcloningkit(Invitrogen, Groningen, TheNetherlands)accordingtothemanufactur er'sinstruc-tions.

CloningefficiencywascheckedthroughPCRampliWcationofseveralcolonies. Theresultingproductswererun onpolyacrylamidegels, withtheinitialgenomicDNAamplificationoutcomeascontrol.Theresolutionusedatthisstepwasespeciallyimportanttodistinguisht hetwoallelesfromheterozygousindividu-als.

AfterplasmidDNApurification(Sambrooketal.1989),th eclonedPCRfragmentsweresequencedbyMWG-

Biotech(http://www.mwgbiotech.com/html/all/index.php). Thesequencedatahavebe ensubmittedtoGenBankunderaccessionnumbersDQ7873 68-

DQ787384.SequenceswereeditedandanalyzedusingtheD NASTARsoftwarepackage(Lasergene, Madi-

son,WI,USA).

Dataanalysis

Numberofalleles,observedheterozygosity(Ho),genedivers 
informationcontent(PIC), nullallelefrequency(rb)andh eterozygotedeficiencyorexcess(Fis)werecalculatedforeachlocusandforthefourspeciesrepresentedbymorethanfivegenotypes, asindicatedinPoncet etal.(2004), usingPowerMarker(LiuandMuse2005).Fo reachspecies,polymorphiclociat0.05thresholdfrequen cyandmeanallelenumberperpolymorphiclocuswereevaluated.

\section{Results}

ESTunigenesets

Atotalof9,820(94\%)validESTswereobtainedfromallC offea

canephorachromatogramfiles.Theaveragelengthwas6 02bp.Afterclusteringandassembly,5,534unigeneswer eidentified,including3,747singletonsand1,787contigs. TheBLASTresultsrevealedthatabout $22.3 \%$ oftheseNR ESTsshowednosimilaritytoanyGenBanksequences.Le ssthan $0.03 \%$ oftheESTswaspredictedtobefromplastid cDNA.

FrequencyanddistributionofC.canephoraEST-SSR

EST-

SSRswereminedfromaNRESTsetof5,534potentialunige nes.Accordingtothesearchcriteriaadopted,431uniqueSS Rswerefoundwithinatotalof376unigenes.Thechanceoffi ndinganSSR-

containingsequenceintheNRESTdatabasewasthus6.8\%, withanaveragedensityofonemicrosatelliteevery $7.73 \mathrm{~kb}$.

Trinucleotidemotifswerethemostabundant(34.34\%), foll owedbydi-(25.75\%), andhexa-nucleotiderepeats(22.04\%)(Fig.2).Themostabundanttrinucleotiderepeatmotifwas AGG/TCC(23\%),followedbyAAG/TTC(20.3\%)andAAC/TTGandAAT/T TAweretheleastabundantmotifs(3.4\% each)(Fig.2).A mongthedinucleotidemotifs,GA/CTwasthemostabundant $(62.2 \%$ )andnoGC/CGmotifwasfound.Forallrepeatclasses, the meanSSRlengthwas

20.6bp,butwashigherfordinucleotides(24.1bp), with amaximum of $94 \mathrm{bp}$.

Outofthe 25EST-

SSRsusedfordesigningprimers, ninewerefoundintransl atedregions, eightinthe 5 untranslatedterminalregion $(5$ UTR)andfourinthe3UTR.Thefourothersequencesgen eratednohitsinthesimilaritysearch(Table1).

ConservationoforthologousSSRlociamong

Coffea species
ToassesstheconservationofC.canephoramicrosatellitelociacrossCoffea species, we testedthe 


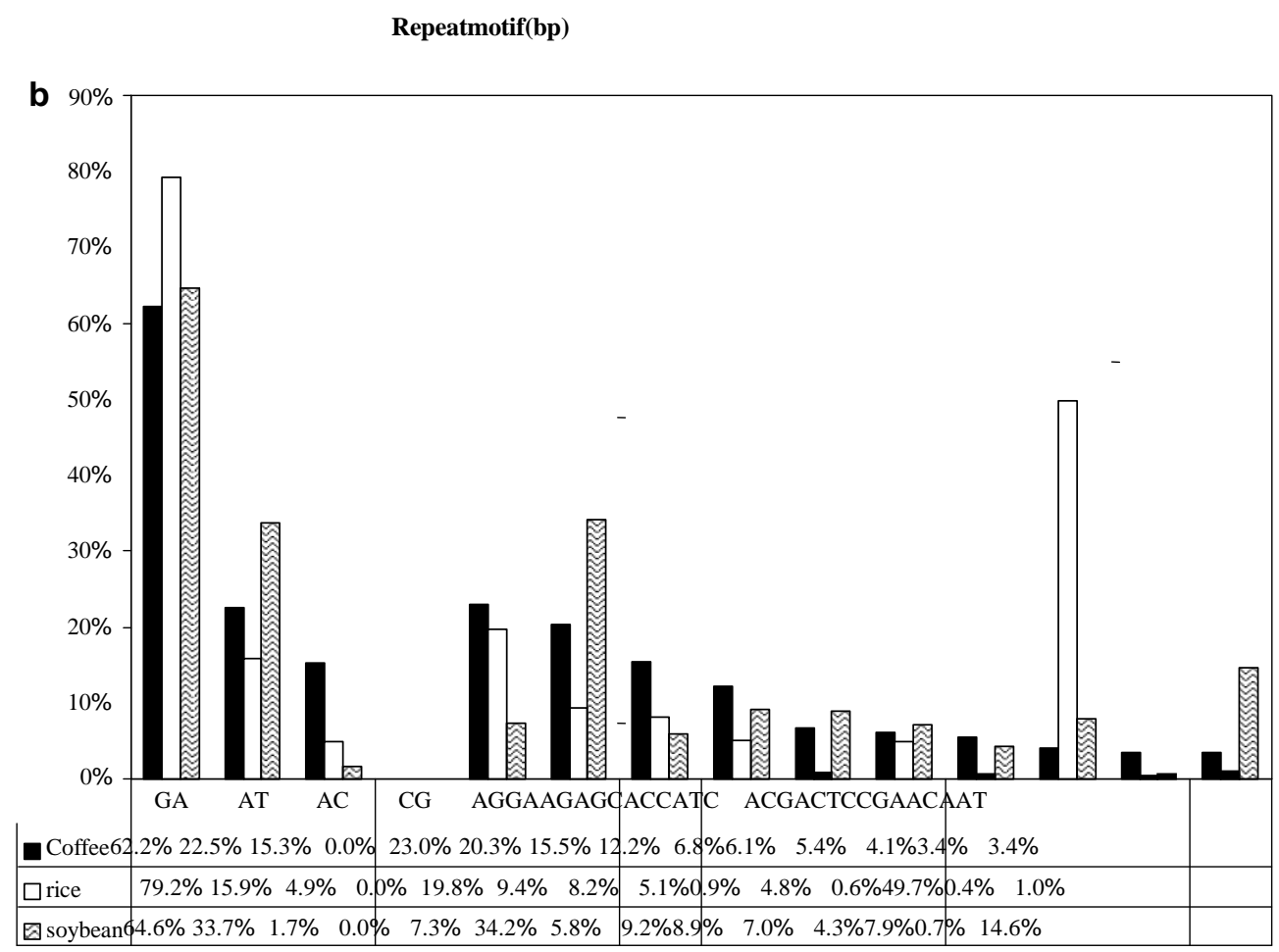

Fig.2 SSRfrequencyintheunigene Coffea

canephoraESTset(riceandsoybeandatafromGaoetal.2003)aaccordingtotherepeatmotiflength,baccordingtothemotifofdiandtrinucleotiderepeats

cross-amplificationof 25primerpairsonsevenspeciesrepresentingthreedifferent $C$ offea phylogeneticclades. Out of 25, 24primerpairs amplified atleastonespecies(onlyES21couldnot)(Table2).Twomicrosatellites(ES3,ES15)did notamplifywell,and theamplifiedproductswere substantiallylargerthanexpected. Finally,crossspeciestransferabilitywithintheCoffea genuswasveryhighsinceeachprimerpairyieldedaPCRprod uctinanaverageof6.1species(outofseventested)and14(56 $\%)$ gaveamplificationwith alltestedCoffea species.

Oneortwobandspergenotypewereobservedinallbutone ofthereadableamplificationpatternsobtained,suggestingthat therewas ofonelocus.TheES11 primerpairamplified36productswithsome C.libericagenotypes,indicatingapossible selectiveamplification 
triplicatesequence-

thiswasconsideredasmissingdataforthesubse quentanalyses.

Giventhatprimersweredesignedonthebasisof

C. canephoraESTsequences, ampliconsproducedfromC.canephoragenomi cDNAhad

expectedsizeinmostcases(Table2),consistent

withlocus-spe-

cificamplification.InallotherCoffea

species, theallelesizesalsodidnotsubstantially

differfromtheexpectedsize, whichwasconsiste ntwithselectiveorthologousamplification.The microsatelliteallelesizedistribu-tions,

expressedinbase pairs, fittedthe stepwisemodelinmostcases(Table 2)withallelicdifferences, whicharemultiplesofth erepeatmotifevenifsomeintermediateallelicstatesweremissing.Some"jumps"i nallelesizedistribution, associatedwithmixed distribu-tionpatterns, mightbeattributed to theadditional 
Table1

Expressedsequencetag(EST),putativegenefunctionbasedontBLASTxsearchofGenBanksequences(analysisonDecember2005),SSRpositioninthecDNAsequence(correspondingrepeateda minoacidwhentranslated)

\begin{tabular}{|c|c|c|c|c|c|c|c|c|}
\hline \multicolumn{4}{|c|}{$\overline{\text { Coffea } \text { EST-SSR }}$} & \multicolumn{5}{|c|}{ Homologous sequences } \\
\hline Locus & Repeatmotif & $\begin{array}{l}\text { Expected } \\
\text { ampliWcon } \\
\text { size(bp) }\end{array}$ & SSRposition & $\begin{array}{l}\text { GenBank } \\
\text { accession } \\
\text { No. }\end{array}$ & Species & $\begin{array}{l}\text { BLAST } \\
\text { Evalue }\end{array}$ & $\begin{array}{l}\text { Identity } \\
(\%)\end{array}$ & Putativefunction \\
\hline ES1 & $(\mathrm{GCA})_{8}$ & 308 & ORF(Q) & AAD02552 & Petuniahybrida & $2 e_{j} 06$ & 64 & PGPS(P-glycoprotein) \\
\hline ES2 & $(\mathrm{A})_{14}(\mathrm{CA})_{19}$ & 349 & & & & & & Nohit \\
\hline ES3 & $(\text { TTTCT })_{9}$ & 371 & 5 UTR & CAB75429 & $\begin{array}{l}\text { Nicotiana } \\
\text { plumbaginifolia }\end{array}$ & $1 \mathrm{e}_{\mathrm{j}} 41$ & 90 & $\begin{array}{l}\text { Oligouridylate } \\
\text { bindingprotein }\end{array}$ \\
\hline ES4 & $(\mathrm{CT})_{14}$ & 337 & 3 UTR & CAA64545 & Hordeumvulgare & $4 e_{j} 21$ & 73 & Xyloseisomerase \\
\hline ES5 & $(\mathrm{TTC})_{24}$ & 309 & ORF (S) & AAL65125 & Glycinemax & $2 e_{j} 31$ & 66 & GT-2transcriptionfactor \\
\hline ES6 & $(\mathrm{CCA})_{2}(\mathrm{CCT})_{11}$ & 228 & 5 UTR & AAN71762 & Solanumtuberosum & $1 \mathrm{e} i 41$ & 75 & CinnamoylCoAreductase2 \\
\hline ES7 & $(\mathrm{TCA})_{18}$ & 298 & & & & & & Nohit \\
\hline ES8 & $(\mathrm{TC})_{26}$ & 323 & 5'UTR & AAB84350 & Arabidopsisthaliana & $3 e \nmid 31$ & 72 & Heatshocktranscriptionfactor \\
\hline ES9 & $(\text { TTC })_{10}$ & 205 & 5 UTR & AAD55726 & Vitisriparia & $7 \mathrm{e} i 74$ & 75 & Galactinolsynthase \\
\hline ES10 & $(\mathrm{ACC})_{11} /(\mathrm{TCC})_{6}$ & 403 & $\mathrm{ORF}(\mathrm{T} / \mathrm{S})$ & AAP40485 & Arabidopsisthaliana & $7 e-47$ & 56 & AP2domaintranscriptionfactor \\
\hline ES11 & $(\mathrm{TTC})_{10}$ & 240 & 5 UTR & AAN28269 & Gossypiumhirsutum & $1 \mathrm{e} ; 63$ & 94 & myb-Liketranscriptionfactor \\
\hline ES12 & $(\mathrm{AAT})_{4}(\mathrm{CAG})_{13}$ & 156 & ORF(Q) & AAM54033 & Populustremula & $8 \mathrm{e} ; 69$ & 67 & PIN1-likeauxintransportprotein \\
\hline ES13 & $(\mathrm{AG})_{16}$ & 235 & 5 UTR & AAW28999 & Antirrhinummajus & $3 e_{i} 44$ & 53 & $\begin{array}{l}\text { 1-Deoxy-D-xylulose-5-phosphate } \\
\text { synthase }\end{array}$ \\
\hline ES14 & $(\mathrm{CCA})_{5} /(\mathrm{CCA})_{10}$ & 289 & $\mathrm{ORF}(\mathrm{T})$ & AAF01534 & Arabidopsisthaliana & $1 \mathrm{e} ; 53$ & 56 & Proteinkinase \\
\hline ES15 & $(\mathrm{CT})_{12}$ & 308 & 5 UTR & AAD42941 & Catharanthusroseus & $2 e_{j} 26$ & 81 & Ubiquitin-conjugatingenzymeE2 \\
\hline ES16 & $(\mathrm{TA})_{15}$ & 186 & 3 UTR & AAC62396 & Ricinuscommunis & $2 e_{j} 54$ & 72 & Cysteineendopeptidaseprecursor \\
\hline ES17 & $(\mathrm{TG})_{16}$ & 300 & 5 UTR & CAG18177 & Arabidopsisthaliana & $1 \mathrm{e} i 74$ & 83 & UDP-galactosetransporter \\
\hline ES18 & $(\text { GGATCA })_{5}$ & 202 & $\mathrm{ORF}(\mathrm{SG})$ & AAM67530 & Arabidopsisthaliana & $1 \mathrm{e} ; 64$ & 58 & Unknownprotein \\
\hline ES19 & $(\mathrm{CA})_{13}(\mathrm{TA})_{6}$ & 259 & $\mathrm{ORF}(\mathrm{TH})$ & AAQ14193 & Solanumtuberosum & $2 \mathrm{e} ; 62$ & 67 & Calciumhomeostasisregulator \\
\hline ES20 & $(\mathrm{AAC})_{9}$ & 197 & $\mathrm{ORF}(\mathrm{N})$ & AAO45753 & Cucumismelo & $1 \mathrm{e} ; 51$ & 57 & $\begin{array}{l}\text { RING/C3HC4/PHD } \\
\text { zincWngerprotein }\end{array}$ \\
\hline ES21 & $(\mathrm{T})_{28}$ & 254 & 3 UTR & AAO33591. & Arachishypogaea & $7 e j 50$ & 65 & Earlylightinducedprotein \\
\hline $\mathrm{ES} 22$ & $\left(\right.$ TTTTTTC $_{4}$ & 207 & 3 UTR & P25317 & Nicotianatabacum & $7 \mathrm{e} i 50$ & 65 & $\begin{array}{l}\text { glutathione } S \text {-transferase } \\
\text { (auxin-regulatedprotein) }\end{array}$ \\
\hline ES23 & $(\mathrm{AG})_{16}$ & 230 & & & & & & Nohit \\
\hline ES24 & $(\mathrm{TCTCC})_{7}$ & 224 & & & & & & Nohit \\
\hline ES25 & $(\mathrm{CCT})_{9}$ & 207 & $\mathrm{ORF}(\mathrm{P})$ & AAG45420 & Chlamydomonasreinhardtii & $6 e_{j} 10$ & 33 & Vegetativecell wall proteingp1 \\
\hline
\end{tabular}


Table2 Numberandmolecularsizerangesof25EST-SSRtestedoneightCoffea species:C.canephoraCAN,C.dewevreiDEW,C.pseudozanguebariaePSE,C.libericaLIB, C. heterocalyxHET,C.eugenioides EUG, and C.sp.MoloundouMOL(samplesizegiveninbrackets)

\begin{tabular}{|c|c|c|c|c|c|c|c|c|c|c|}
\hline \multirow[t]{2}{*}{ EST-SSR } & \multirow{2}{*}{$\begin{array}{c}\text { Expecte } \\
\text { dsize(bp) }\end{array}$} & \multicolumn{7}{|c|}{ Allelesizerange $(\mathrm{bp}) /$ alleleno. } & \multirow{2}{*}{$\begin{array}{l}\text { Totalno } \\
\text { ofalleles }\end{array}$} & \multirow{2}{*}{$\begin{array}{l}\text { Typeofallelesized } \\
\text { stribution }\end{array}$} \\
\hline & & CAN $\left(I^{\prime} 2\right)$ & DEW (IU) & $\operatorname{PSE}(10)$ & $\operatorname{LIB}(\gamma)$ & HEII(1) & $\operatorname{EUG}(2)$ & MUL(2) & & \\
\hline ES1 & 327 & $\begin{array}{l}318-327 \\
3\end{array}$ & $306-318$ & $306-321$ & $306-318$ & 318 & 318 & 327 & 7 & Stepwise \\
\hline \multirow[t]{2}{*}{ ES2 } & 368 & $356-371$ & $356-363$ & $342-356$ & $342-358$ & 342 & 346 & 349 & & Stepwise \\
\hline & & 6 & 4 & 3 & 4 & 1 & 1 & 1 & 10 & \\
\hline \multirow[t]{2}{*}{ ES3 } & 390 & $>2,000$ & $>2,000$ & $>2,000$ & $>2,000$ & $>2,000$ & $>2,000$ & $>2,000$ & & - \\
\hline & & - & _- & - & - & - & - & - & & \\
\hline \multirow[t]{2}{*}{ ES4 } & 356 & $344-372$ & $338-396$ & $338-362$ & $346-372$ & 350 & $362-368$ & - & & Mixed \\
\hline & & 8 & 8 & 5 & 6 & 1 & 3 & - & 15 & \\
\hline \multirow[t]{2}{*}{ ES5 } & 328 & $309-315$ & $291-306$ & $300-309$ & $297-315$ & 294 & $291-294$ & 300 & & Stepwise \\
\hline & & 3 & 5 & 4 & 4 & 1 & 2 & 1 & 9 & \\
\hline \multirow[t]{2}{*}{ ES6 } & 247 & 256 & $238-268$ & $244-259$ & $256-298$ & $238-256$ & $238-256$ & $262-259$ & & Mixed \\
\hline & & 1 & 4 & 3 & 4 & 2 & 3 & 2 & 9 & \\
\hline \multirow[t]{2}{*}{ ES7 } & 317 & $300-324$ & $302-315$ & $288-290$ & $288-307$ & 307 & $288-302$ & 332 & & Nearlycontinuous \\
\hline & & 10 & 6 & 2 & 6 & 1 & 3 & 1 & 19 & \\
\hline \multirow[t]{2}{*}{ ES8 } & 251 & $236-248$ & $246-258$ & $222-234$ & $236-258$ & 228 & $226-234$ & 224 & & Stepwise \\
\hline & & 5 & 4 & 3 & 5 & 1 & 2 & 1 & 13 & \\
\hline \multirow[t]{2}{*}{ ES9 } & 224 & $204-230$ & $210-218$ & 214 & 204-214 & - & 215 & - & & Nearlystepwise \\
\hline & & 6 & 3 & 1 & 4 & - & 1 & - & 9 & \\
\hline \multirow[t]{2}{*}{ ES10 } & 422 & $395-415$ & $392-420$ & 404 & $389-404$ & - & - & - & & Nearlystepwise \\
\hline & & 7 & 5 & 1 & 5 & - & - & - & 11 & \\
\hline \multirow[t]{2}{*}{ ES11 } & 259 & $246-266$ & $240-269$ & $254-260$ & $240-266$ & 246 & 234 & $243-263$ & & Nearlystepwise \\
\hline & & 8 & 6 & 2 & 9 & 1 & 2 & 2 & 14 & \\
\hline \multirow[t]{2}{*}{ ES12 } & 175 & $160-178$ & $169-178$ & $151-166$ & $169-178$ & 154 & $160-163$ & 169 & & Stepwise \\
\hline & & 5 & 3 & 2 & 4 & 1 & 2 & 1 & 9 & \\
\hline \multirow[t]{2}{*}{ ES13 } & 254 & $235-255$ & $237-241$ & $233-237$ & $233-241$ & 237 & $239-241$ & - & & Stepwise \\
\hline & & 10 & 2 & 2 & 2 & 1 & 2 & - & 12 & \\
\hline \multirow[t]{2}{*}{ ES14 } & 308 & 297 & $291-297$ & 294-297 & 291-294 & 297 & - & 297 & & Stepwise \\
\hline & & 1 & 3 & 2 & 2 & 1 & - & 1 & 3 & \\
\hline ES15 & 327 & & & & Nonscorable & & & & & - \\
\hline \multirow[t]{2}{*}{ ES16 } & 205 & $180-222$ & 206-212 & $195-212$ & $207-236$ & - & 196-198 & 196 & & Mixed \\
\hline & & 11 & 4 & 2 & 8 & - & 2 & 1 & 17 & \\
\hline ES17 & 319 & $\gg 640$ & $\gg 640$ & $\gg 640$ & $\gg 640$ & $\gg 640$ & $\gg 640$ & $\gg 640$ & & \\
\hline \multirow[t]{2}{*}{ ES18 } & 221 & $218-222$ & 212 & $206-218$ & $212-218$ & 206 & 206 & $206-218$ & & Stepwise \\
\hline & & 2 & 1 & 2 & 2 & 1 & 1 & 1 & 4 & \\
\hline \multirow[t]{2}{*}{ ES19 } & 278 & $256-284$ & 250 & $250-252$ & 244 & 252 & 252 & 242 & & Stepwise \\
\hline & & 9 & 1 & 2 & 1 & 1 & 1 & 1 & 13 & \\
\hline ES20 & 216 & 204-219 & 212 & $215-229$ & $209-212$ & 212 & $212-219$ & 212 & & Mixed \\
\hline & & 4 & 1 & 7 & 2 & 1 & 2 & 1 & 11 & \\
\hline ES21 & 273 & - & - & - & - & - & - & - & 0 & - \\
\hline ES22 & 226 & $213-232$ & - & - & $213-220$ & - & 230 & - & & Mixed \\
\hline & & 6 & - & - & 2 & - & 1 & - & 6 & \\
\hline ES23 & 249 & $236-262$ & $238-240$ & $240-270$ & $236-260$ & 246 & 236 & $244-246$ & & Stepwise \\
\hline & & 8 & 2 & 12 & 5 & 1 & 1 & 2 & 16 & \\
\hline
\end{tabular}


contributionofinsertions/deletions(indels)intheamplifie dregion. Thispatternwasobservedbetweenspeciesatthe ES16locus.However,somesubstantialallelesizedifferen ceswereobservedwiththreeprimerpairs(ES3,ES15andE S17), withampliconsmuchlargerthanexpected, irrespect iveofthespecies.Forinstance, theobservedallelesizeforE S17wasabout640bpforallspeciesversus319bpexpected. ProductsobtainedbyamplificationwithES2,ES4,ES6,E S16,ES17,ES19, andES25primerpairswereclonedandsequ encedtoconfirmtheorthology.Theywerealsoanalyzedtoide ntifytheoriginofwithin-andbetween-

speciespolymorphismas

wellasdiscrepanciesbetweentheobservedandexpectedallel esizes(Table3).Thegenotypeschosenforcloningpresented allelesizesattheextremesofthedistributionofagivenmarker. Therelativesequencesizeswereinagreementwiththesizese valuatedaftermigration, withameanSAGAsoftwareundere stimationof

¡6.72bp.ThegenomicDNAsequencesclarifiedthreemainpo ints:

(1) therewasstrongsequencehomologyallalongtheregions flankingtherepeatmotifbetweenampliconsderivedfromdif ferentspecies(mean96.9\%identity),inagreementwiththea mplificationoforthologousloci;(2)theobservedpolymorph ismmainlyresultedfromvariationsinrepeatnumber,althoug hsomemorecomplexmutationpatterns(ES6,ES16)wereals odetected,involvingadditionalvariationofindelsintheflank ingsequences;(3)thelargesizeofES17genomicfragmentsw asduetoamplificationofa426bpintroninallspecies,althoug hthepolymorphismstillresultedfromadiVerentnumberofre peats

Within-andbetween-speciespolymorphism

Thenumberandmolecularsizerangesofallelesobtainedwi theachEST-

SSRareshowninTable2togetherwiththenumberofpolym orphicloci.OnelocusappearedtobemonomorphicforC.li berica(ES19),twoforC.canephora(ES6,ES14)and

C.pseudozanguebariae(ES9,ES10), and threefor C.dewevrei(ES18,ES19,ES20).Fortheremaining loci,upto12allelesperlocuswererecorded(ES23amplificationinC.pseudozanguebariae).Forthethreespeciesrepres entedbyone (C.heterocalyx), two(C.sp.Moloundou)orthree(C.eugenioides)genotypes, 18allelesat 17 and 15lociweredetectedforC.heterocalyxandC.sp. Moloundou, respectively, while33allelesat19lociwereobservedforC.eu genioides.

Whenconsideringmarkerswithdi-, tri, andmoremicrosatelliterepeatedmotifs, theaveragenumber ofallelesdetectedperlocuswas 14.3(86alleles,6loci), 9.7(107alleles,11loci)and5.7(17alleles,3loci), 
Table3AllelesequenceanalysesatsevenmicrosatellitelocifordiVeren tspeciesgenotypes(homozygotesorheterozygotes)rela-tivetothe C.canephoraESTsequence(ESTdb):total length,

\begin{tabular}{|c|c|c|c|c|c|c|}
\hline Locus & Coffea species & Individual & Cloneno. & $\operatorname{Size}(b p)$ & Polymorphism & $\begin{array}{l}\text { Percentagesimilarityo } \\
\text { fXankingsequences }\end{array}$ \\
\hline \multirow[t]{4}{*}{ ES2 } & CAN & $E S T d b$ & & 349 & $(\mathrm{~A})_{14}(\mathrm{CA})_{19}$ & 100 \\
\hline & PSE & P-8056 & ES2-11 & 341 & $(\mathrm{~A})_{14}(\mathrm{CA})_{15}$ & 97.3 \\
\hline & CAN & C-IF182 & ES2-21 & 357 & $(\mathrm{~A})_{18}(\mathrm{CA})_{21}$ & 98.0 \\
\hline & & & ES2-22 & 351 & $(\mathrm{~A})_{18}(\mathrm{CA})_{18}$ & 98.3 \\
\hline \multirow[t]{2}{*}{ ES4 } & CAN & $E S T d b$ & & 337 & $(\mathrm{CT})_{14}$ & 100 \\
\hline & DEW & D5765 & ES4-15 & 349 & $(\mathrm{CT})_{20}$ & 97.4 \\
\hline \multirow[t]{5}{*}{ ES6 } & CAN & $E S T d b$ & & 228 & $(\mathrm{CCA})_{2}(\mathrm{CCT})_{11}$ & 100 \\
\hline & PSE & $\mathrm{P}-55$ & ES6-12 & 227 & $(\mathrm{CCA})_{3}(\mathrm{CCT})_{8}$ & 95.5 \\
\hline & & & ES6-11 & 220 & $(\mathrm{CCA})_{3}(\mathrm{CCT})_{6}$ & 95.5 \\
\hline & LIB & $\mathrm{L}-\mathrm{A} 12$ & ES6-25 & 255 & $(\mathrm{CCA})_{4}(\mathrm{CCT})_{10}+20 \mathrm{bp}$ & 93.9 \\
\hline & & & ES6-26 & 247 & $(\mathrm{CCA})_{2}(\mathrm{CCT})_{10}$ & 95.5 \\
\hline \multirow[t]{4}{*}{ ES16 } & $\mathrm{CAN}$ & $E S T d b$ & & 186 & $(\mathrm{TA})_{15}$ & 100 \\
\hline & $\mathrm{CAN}$ & C-IF72 & ES16-12 & 199 & $(\mathrm{TA})_{21}$ & 96.1 \\
\hline & & & ES16-25 & 165 & $(\mathrm{TA})_{4}$ & 95.9 \\
\hline & LIB & L-A22 & ES16-15 & 206 & $(\mathrm{TA})_{17}+17 \mathrm{bp}$ & 95.5 \\
\hline \multirow[t]{2}{*}{ ES17 } & CAN & $E S T d b$ & & 300 & $(\mathrm{TG})_{16}$ & 100 \\
\hline & PSE & P60 & ES17-10 & 718 & $(\mathrm{TG})_{10}+$ intron $426 \mathrm{pb}$ & 97.9 \\
\hline \multirow[t]{4}{*}{ ES19 } & $\mathrm{CAN}$ & $E S T d b$ & & 260 & $(\mathrm{CA})_{13}(\mathrm{TA})_{6}(\mathrm{~T})_{2}$ & 100 \\
\hline & $\mathrm{CAN}$ & C-IF461 & ES19-15 & 266 & $(\mathrm{CA})_{10}(\mathrm{TA})_{11}(\mathrm{~T})_{4}$ & 98.6 \\
\hline & & & ES19-16 & 246 & $(\mathrm{CA})_{2}(\mathrm{TA})_{7}(\mathrm{~T})_{9}$ & 98.2 \\
\hline & LIB & L-A11 & ES19-26 & 233 & $(\mathrm{CA})_{1}(\mathrm{TA})_{0}(\mathrm{~T})_{9}$ & 99.1 \\
\hline \multirow[t]{3}{*}{$\mathrm{ES} 25$} & $\mathrm{CAN}$ & $E S T d b$ & & 207 & $(\mathrm{CCT})_{9}$ & 100 \\
\hline & DEW & D-5462 & ES25-11 & 200 & $(\mathrm{CCT})_{6}$ & 98.3 \\
\hline & PSE & P-1 & $\mathrm{ES} 25-24$ & 219 & $(\mathrm{CCT})_{13}$ & 96.0 \\
\hline
\end{tabular}

microsatelliterepeat stretches, introns, indels(insertions/deletions), and\%similarityoftheXankingsequences

CANC.canephora,DEWC.dewevrei,PSEC.pseudozanguebariae,LIBC.liberica

respectively.Whenconsideringthelocationofthemicrosatel litemotifinrelationwiththegeneannota-

tion, anaverageof8.1(73/9),12.7(11/3), and11.4(57/

5)allelesperlocuswereobtainedforORF,3UTRand5UTRlo cations, respectively.

Clearlyreadablelociwereanalyzedtoevaluategeneticdiver sityparameters, whiletakingintoaccountonlysin-gle-

locusmarkers which gave anamplification withmorethanfivegenotypesperspecies, between16(C.liberic a)to21(C.canephora)(Table4).Ahighlevelofpolymorphiclociwasobservedwhateverthespeciesconsidered(from75 $\%$ (C.liberica)to $86 \%$ (C.canephora)). Themeanallelenumbe rperpolymorphiclocuswashighestforC.canephora(6.4)wh ilelowerbutsimilarvalueswereobtainedforC.dewevrei,

C.liberica, and

C.pseudozanguebariae (4.1,4.1, and3.5, respectively).The observed heterozygosity was lowest for C.pseudozanguebariae (0.28)andhighestforC.libericaandC .canephora(0.52and0.51,respectively).ThePICvaluerang edfrom0(monomorphlocus)to0.91(ES23forC.pseudozang uebariae, highlypolymorphiclocus)acrossthefourspecies, withthelowestaveragevalueobtainedforC.pseudozangueb ariae (0.40)andsimilaraveragevaluesforC.canephoraandC .liberica
(0.59and0.57).Therewasnosignificantcorrelationbetweeni nterspecificPICvaluesexcept forthe

C.liberica/C.dewevreicombination $(0.78 ; P=0.003)$.

Althoughvariationsbetweenlociwereobserved, aglobal heterozygotedeficit(Fis $=0.10$

$0.26)$ andthepresenceofnullalleles $(\mathrm{rb}=$ $0.03-$ 0.09)couldbeesti-

matedforallspecies. Thelowestvalueswereobtainedfor C.ca nephoraand $\quad C$. liberica, whilethe highestwereobtainedforC.dewevreiandC.pseudozangueb ariae.

DiagnosticallelesandlocusidentiWcation

Allelesizesidentifiedforthecultivatedspecies

C.canephora $(\mathrm{CAN})$ werecomparedtotheothers.DEW/PSE speciescomparisonwasalsoconsideredsincethiscouplewasi nvolvedinthefirstinterspecificmap(Kyetal.2000).From4to1 6locicouldbeidenti-

fiedasdiagnosticmarkers,i.e.markersforwhichtherewereno sharedallelesbetweenspecies(Table4).Thesmallestnumber ofsuchlociwas

obtainedfortheCAN/LIBcomparisonandthegreatestforCA N/MOL.Moreover,withinthesemarkers,somewerecharact 
Table4 Diversitystatisticsfor21EST-SSRinCoffea speciesasdescribedbytheexpected(He)andobserved(Ho)heterozygosities, thepolymorphisminformationcontent(PIC),nullallelefrequency(rb), andheterozygotedeWciencyorexcess(Fis)

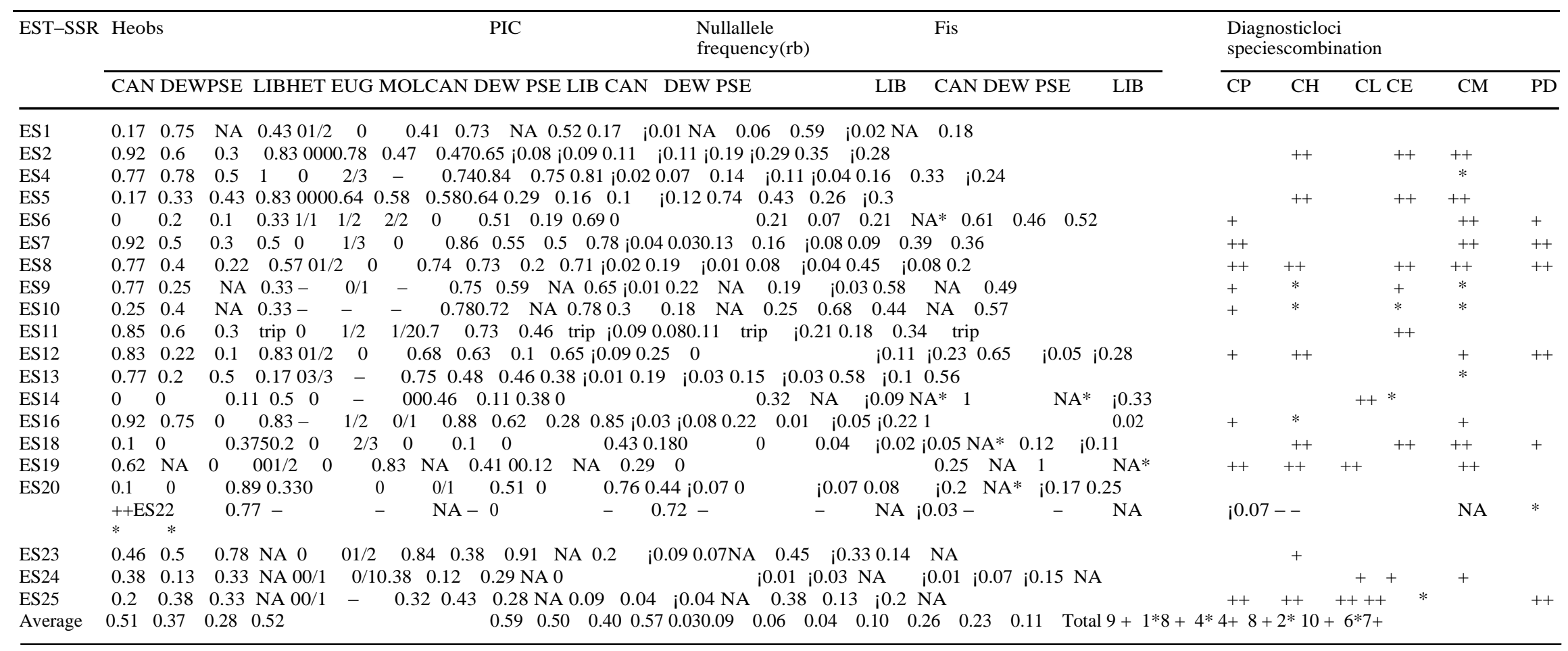

Diagnosticloci: withnooverlappingallelicrangebetweenspeciesofeachcouple

NAnotapplicable(lessthanWveindividualsanalysedor divisionbyzero),-indicatesnoamplification,tripdenotestriplicatedlocus, ++indicatesnooverlappingallelesizedistributions, +indicatesnocommonallelesbetweenthetwospecies, *indicatesnoampliWcationwiththenon-

CANspecies,C:C.canephora,P:C.pseudozanguebariae,H:C.heterocalyx, E:C.eugenioides, M:C.sp.Moloundou,D:C.dewevrei,L:C.liberica 
threeforCAN/LIB,sixforCAN/EUG,sevenforCAN/MOL, and $W_{v e}$ for PSE/DEWcomparisons.

\section{Discussion}

\section{ESTsarearichsourceofSSRsinCoffea}

Atotalof431uniqueSSRswereidentiWedfrom5,534pote ntialunigenes. Theseresultsclearlydemonstratedthat $C o f$ fea ESTsareavaluableresourceforminingSSRmarkers.

RandomsequencingwithincDNAlibrariesleadstoahig hproportionofredundantESTs.Inourstudy,redun-

dancywaseliminatedpriortoanalysisinordertoreducethed atasetsize.TheadvantageofusingNRESTsistoavoidovere stimationoftheEST-

SSRfrequency.Forexample, afterredundancyelimination, KumpatlaandMukhopadhyay(2005)observed37.3\%loss inthenum-berofSSR-ESTforArabidopsisthaliana.

Forcoffeetrees, wefoundthat $6.8 \%$ potentialunigenescontainedmicrosatellitemotifs, withanaverageofon emicrosatelliteevery $7.73 \mathrm{kbofEST}$ sequence.ThisSSRESTfrequencywasinthe2.65-

$10.62 \%$ rangeobtainedbyKumpatlaandMukhopadhyay (2005)fordicotspecies.Itwasslightlyhigherthanthe 1.54.7\% rangereportedbyKantetyetal.(2002)formonocots. Theoverallfrequencyandthe frequencyofthediVerentrepeatmotifsareknowntobedep endentonthepresence ornotofredundancy,butalsorelatedtothecriteriausedtoid entifySSRinthedatabasemin-

ing.Ingeneral,whentheminimumrepeatlengthis $20 \mathrm{bp}, \mathrm{mi}$ crosatellitesofvariousplantspeciesarepresentinabout5\%ofESTs(Varshneyetal.2005a).

OurresultsweresomewhatinagreementwiththisestimationalthoughouroverallSSR-

ESTfrequencymighthavebeen

inXatedbythedetectionofhexanucleodiderepeatmotifsi ntheanalysis, afactorthatisseldomconsideredinotherstud ies(seebelow).

InC.canephoraESTs, trinucleotiderepeatswerefound tobethemostabundant(34.34\%),followedbydi$(25.75 \%)$ andhexa-nucleotidemotifs $(22.04 \%)$.

Trinucleotiderepeatsaregenerallythemostcommonmo tiffoundinbothmonocots(54-

78\%amongcereals, Varshneyetal.2005a)anddicots(forex ample51.5\% inMedicagotruncatula,Eujayletal.2004).On lyonereport(KumpatlaandMukhopadhyay2005)hasdesc ribedtheprevalenceofdinucleotiderepeatsinmostofthedic otsinvestigated.However, theysuggestedthattheirresultm ighthavebeenduetotheover-representa-

tionofuntranslatedregions(UTRs)comparedwithopenrea dingframes(ORFs).Indeed,becauseoftheabsenceofframe shiftmutationswhentherearelength variationsintri-

andhexanucleotiderepeats, thesemotifsarefoundinexcess inbothcodingandnoncodingsequences, butotherrepeatty pesaremuchlessfrequentincodingregionsthaninUTRs(M etzgaretal.2000).Ourresultsconfirmedthisdistribution(Ta ble1):7/9microsatellitesfoundincodingsequencesweretri nu-

cleotiderepeatsandonewasanhexanucleotiderepeat, wher easmicrosatellitesinUTRweremainlydinucleotiderepeats(6/12)andtrinucleotiderepeats(3/12).

Asmostmicrosatellitelibrariesusedformarkerdevelop mentaregenerallyenrichedindi-,tri-andtetranucleotiderepeats,computationalminingofESTdatabase smainlyinvolvesthesetypesofmotifs(Kantetyetal.2002;Morganteetal.2002;Eujayletal.2004;Pint oetal.2004;LaRotaetal.2005).However,anon-

negligibleabundanceofmono-

andhexanucleotideSSRswasobservedinourstudy,i.e.12. 30and $22.04 \%$,respectively(Fig.2). Thesedatawereinclo seagree-

mentwiththoseofGao(2003), andsupportedtheresultsobt ainedbyKumpatlaandMukhopadhyay(2005)andMorga nteetal.(2002)ontheabundanceofmononucleotideSSRs. Moreover,whenconsideringCoffea

microsatelliteswithmono-

tohexanucleotiderepeats, theirrelativedistributionmatch edthatnotedinsoybean(Gaoetal.2003;Fig.2).

TheGA/CTmotifwasthemostabundantdinucleotidemotif(62.2\%)inourCoffea

ESTs.Thesemotifswerealso themostfrequentlyobservedSSRs in plants(Scottetal.2000;Gaoetal.2003;Thieletal.2003;Sah aetal.2004).Themostabundanttri-

nucleotiderepeatmotifdetectedinthepresentstudywasA GG/TCC(23\%), closelyfollowed by AAG/TTC (20.3\%).Theseresultsareinagreementwithotherreportso ndicotspe-

cies(Scottetal.2000;Eujayletal.2004;KumpatlaandMuk hopadhyay2005).TherareCCG/GGCfrequencycompare dwithrice(Fig.2)conWrmedthegeneraltrendsnotedinmo nocots,i.e.theyhave moreCGrichtrinucleotiderepeatsthandicots(Morganteetal.2002).

OrthologampliWcationandcross-

speciestransferability

OurcomputationalstrategytodetectNRSSRsanddevelop uniqueEST-

SSRmarkersappearedeYcientsincenearlyallthemarkers appearedtobesinglelocusspeciWc.

Theuse ofa set ofNRsequenceswas important forthedevelopmentofuniquegenicSSRmarkers. Thespec iWcityofthedesignedprimerpairswasthencheckedbyblast ingagainsttheESTsequences.However,thepresenceofpar alogs(sequencesderivedfromduplica- 
omacommonancestor), mightstillbepossibleand itesanalyzedonfourCoffea

species(2to9alleles).Acomparisonofgeneticparametersesti matedfromthispreviousstudy(Poncetetal.2004)andthe thesourceofdiffcultiesininterpreting betweenspeciescomparisons. The $>94 \%$ identityofampliconsequen cesinSSRflankingregionsandthemaintenanceofrepeatmotifsconfirmedthecrossspeciessequenceconservationandtheprimerspecificity.Fin ally, theorthologywasfurtherconfirmedbyananalysisofamp lificationpatterns(sizeandnumb"erofamplificationproduct s).

Cross-speciestransferabilitywithintheCoffea genuswasveryhighsinceeachprimerpairyieldedaPCRprod uctinanaverageof6.1species(outofseventested)and14(56 $\%)$ gaveamplificationwithallthetestedCoffea species,regardlessofthephylogeneticrelationships.Thisisa nimportantfeatureofgenicSSRmarkers, whicharetransfera bleamong distantlyrelatedspeciesorevengenera(Decroocqetal.2003; Liewlaksaneeyanawinetal.2004;Varshneyetal.2005b;Set hyetal.2006).Comparedtopreviousresultsbasedongenomi cmicrosatellites(Poncetetal.2004),EST-

SSRappearedtobemoretransportablemarkers(62.592vs.61.7\%).Similarly,thetransferabilityofESTSSRmarkersfromPinustaeda(loblollypine)to P.contortassp. latifoliawastotal,whileitwasonly lessthanathirdfornon-ESTderivedmicrosatellitemarkers(Liewlaksaneeyanawinetal.2004).

EST-SSRpolymorphism

Ahighlevelofpolymorphiclociwasnotedwhateverthespeci esconsidered.AlthoughtheconservednatureofESTSSRpromotedtransferability, itcouldalsohavelimitedpoly morphism.Thishasbeensuggestedinseveralreportswheret helevelofEST-SSRpolymor-

phismwaslowerthanthatwithSSRderivedfromgenomiclibr aries(Choet al.2000;Eujaylet al.2001;Guptaetal.2003).However,somerecentstudiesrep ortedhighlevelsofpolymorphismwithEST-

SRRmarkers(Eujayletal. 2004;Fraseretal.2004; Sahaet al.2004), withcaseswhereEST-

SSRmarkerswereassociated withequivalentorevenhigherl evelsofpolymorphismthangenomicSSR(e.g.Liewlaksane e-yanawinetal.2004; Varshneyet al.2005a).OurresultsonsevenCoffea speciesalsosupportedtheseobservations.Threeto19allelesperpolymorphiclocuswererecorded amongthesetofspeciestested.Thisrangeislargerthanthatrep ortedbyBhatetal.(2005)—-7-

13allelesdetectedusingnineEST-SSR - although awiderspectrumofspecieswastested(14Coffea andfourPsilanthus).Interestingly,itisalsolargerthanthatnot edbyPoncetetal.(2004), witharound60genomicmicrosatell 
presentresultscouldbeillustratedwithtwoexamples.First,t hecultivatedspeciesC.canephoradisplayedhighervalues withEST-SSRthanwithgenomicmicro-satellites, i.e.mean allele numberperlocus $\quad(5.7$ vs.3.6), observedheterozygosity(0.51vs. 0.38$) \operatorname{andPIC}(0$. 59vs.0.48).Secondly,comparablevaluesforthesethreepar ametersandforthemeanFisvaluewereobtainedforC.pseu dozanguebariaeindependentlyofthetypeofSSRmarker.

Acomparisonofgeneticparametersobtainedforeachspe cies illustrated thatESTSSRmarkerswereidealforassessinggeneticdiversityincof feetrees.Forexample,C.pseudozanguebariaeappearedto belesspolymorphicthanC.canephorawhenbothgenomic microsatelliteandEST-SSRmarkerswere usedandlesspolymorphicthanC.dewevreiandC.liberica whenusingEST-SSRmarkers.ThisobservationisinagreementwiththemorerestrictedgeographicaldistributionofC.pseudozanguebariae. Whenconsideringthetwore latedspeciesC.libericaandC.dewevrei, theydiVerentiated earlierwhenusingmorphologicaltraits, molecular markers (AFLP) and malefertilityof $F_{1}$ hybrids(N'Diayeetal.2005).TheC. dewevreiindividualsanalyzedinthepresentstudywereformerlycultivatedgenotypescollectedintheCentralAfricanRepublic.T heC.libericasamplecorrespondedto amix ofgeographicoriginscomprisingcultivatedandwildforms. Undertheseconditions, itwasnotsurpris-

ingtoobservehigherlevelsofPICandheterozygosityassoci atedwithlowerFisvalues, andanullallelefre-quency $(\mathrm{rb})$.

AnotherimportantfeatureofourEST-SSRs wastheirefficiencyindistinguishingindividualsfrompairs ofrelatedspecies.40to52\%diagnosticlociwereidentiWedwhentheWveinterspecificcombinations(CAN/EU G, CAN/HET, CAN/LIB, CAN/PSE and PSE/ DEW)wereconsidered.

Finally,ourresultsdemonstratedthat:(1)C.canephoraEST-

SSRmarkerscanbeeasilytransferredtowildCoffea speciesforwhich no informationisavailableontheirDNAsequences;(2)theyaregoodcandidatesforthedevelopmentofconservedorthologousmark ersforgeneticanalysisacross Coffea species.Thishighdegreeoftransferabilitybetweenspeci eswillfacilitatecomparativemappingandhomologousg enecloning.

AcknowledgmentsThisworkwaspartlysupportedbyEUgrantNo.I CA4-CT-2001-10068.TheauthorswishtothankI.Mougenot,C.Fizames,B. Piegu,A.Wissocq,F.Lechauve,F.Moreews, X.Argout,F.Chevalier,andmany GenetropresearchersfortheirinvolvementinthedevelopmentofEST $\mathrm{db}$,andM.LorieuxforhishelpindevelopingtheSSRscript(http://ww w.mpl.ird.fr/bio-info/). ThanktoDr.SantiagoC.González-

Martínezforhisvalu-ablecommentsonthemanuscript. 


\section{References}

AltschulSF,

Madden

TL,

SchaVerAA, ZhangJ,ZhangZ,MillerW,LipmanDJ(1997)GappedBLASTan dPSI-

BLAST:anewgenerationofproteindatabasesearchprograms.Nu cle-icAcidsRes 25:3389-3402

BaruahA,Naik P, Hendre S,Rajkumar R,RajendrakumarP,AggarwalRK(2003)Isolationandcharacteri zationofninemicrosatellitemarkersfrom Coffea

arabicaL.,showingwidecrossspeciesampliWcations.MolEcolNotes3:647-650

BhatPR,KrishnakumarV,HendrePS,RajendrakumarP,VarshneyRK,AggarwalRK(2005)IdentiWcationandcharacterizationofexpressedsequencetagsderivedsimplesequencerepeats, markersfromrobustacoVeeva riety 'CxR'(aninter-speciWchybridofCoffea canephora£Coffea congensis).MolEcolNotes5:80-83

BrownGR,KadelEEIII,BassoniDL,KiehneKL,TemesgenB,van BuijtenenJP,SewellMM,MarshallKA,NealeDB(2001)Anch oredreferencelociinloblollypine(PinustaedaL.)forintegratin gpinegenomics.Genetics 159:799-809

CatoSA,GardnerRC,KentJ,RichardsonTE(2001)ArapidPCRbasedmethodforgeneticallymappingESTs. TheorApplGenet 102:296-306

ChoYG,IshiiT,TemnykhS,ChenX,LipovichL,McCouchSR,Park WD, AyresN,CartinhourS(2000)DiversityofmicrosatellitesderivedfromgenomiclibrariesandGenBanksequencesinrice(OryzasativaL.).TheorApplGenet 100:713-722

CoulibalyI,RevolB,NoirotM,PoncetV,LorieuxM,CarascoLacombe C, Minier J, DufourM, HamonP(2003)AFLPandSSRpolymorphisminaCoffea interspeciWcbackcrossprogeny $[($ C.heterocalyx£C.canephora $) £$ C.canephora $]$.TheorAp plGenet107:1148-1155

DecroocqV, FaveMG, HagenL, BordenaveL,DecroocqS(2003)Developmentandtransferabilityof apricotandgrapeESTmicrosatellitemarkersacrosstaxa.TheorAp plGenet106:912-922

DirlewangerE,CossonP,TavaudM,AranzanaJ,PoizatC,ZanettoA,ArusP,LaigretF(2002)Developmentofmicrosatellitemarkersinpeach[Prunuspersica $($ L.)Batsch]andtheiruse ingenetic diversityanalysisinpeachandsweetcherry(PrunusaviumL.). TheorApplGenet 105:127-138

DufourM,HamonP,NoirotM,RistrerucciAM,BrottierP,VicoV,Lero yT(2001)PotentialuseofSSRmarkersforCoffea spp.geneticmapping.In:ASIC(ed)19thinternationalsciencecolloquiumonco $\mathrm{Vee}$, Trieste,Italy

EllegrenH(2004)Microsatellites:simplesequenceswithcomplexevolution.NatRevGenet5:435-445

EujaylI,SledgeMK,WangL,MayGD,ChekhovskiyK,ZwonitzerJC,MianMA(2004)MedicagotruncatulaEST-

SSRsrevealcross-speciesgeneticmarkersforMedicagospp.TheorApplGenet 108:414-422

EujaylI,SorrellsM,BaumM,WoltersP,Powell W(2001)AssessmentofgenotypicvariationamongcultivateddurumwheatbasedonEST -SSRSandgenomicSSRS.Euphytica119:39-

43FernandezD,SantosP,AgostiniC,BonMC,PetitotAS,SilvaMC, Gu erraGuimaraesL,RibeiroA,ArgoutX,NicoleM(2004)CoVee(Coffea arabica $\mathrm{L}$.)genesearlyexpressedduringinfectionbytherustfungus(Hemileiavastatrix).MolPlantPath ol5:527-536

FraserLG,Harvey CF,Crowhurst RN,De SilvaHN(2004)ESTderivedmicrosatellitesfromActinidiaspeciesandtheirpotentialformapping. TheorApplGenet108:1010-1016

GaoLF,TangJF,LiHW,JiaJZ(2003)Analysisofmicrosatel- litesinmajorcropsassessedbycomputationalandexperimentalapproaches.MolBreed12:245-261 
Gonzalez-MartinezSC,RobledoArnuncioJJ,ColladaC,DiazA,WilliamsCG,AliaR,CerveraMT (2004)Cross-ampliW-cationandsequencevariation ofmicrosatellitelociinEurasianhardpines.TheorApplGenet 109:103-111

GonzaloMJ,OliverM,GarciaMasJ, MonfortA,DolcetSanjuanR,KatzirN,ArusP,MonforteA(2005 )Simple-sequencerepeatmarkersusedinmerginglinkagemapsofmelon $(\mathrm{Cuc}$ umismeloL.).TheorApplGenet110:802-811

GuptaPK,RustgiS,SharmaS,SinghR,KumarN,BalyanHS(2003) TransferableEST-SSRmarkersforthestudyofpolymorphismandgeneticdiversityinbreadwheat.MolGenet Genomics270:315-323

KantetyRV,LaRotaM,MatthewsDE,SorrellsME(2002)Datami ningforsimplesequencerepeatsinexpressedsequencetagsfrom barley,maize,rice,sorghumandwheat.PlantMolBiol48:501510

KumpatlaSP,MukhopadhyayS(2005)Miningandsurveyofsimplesequencerepeatsinexpressedsequencetagsofdicotyledonous species.Genome48:985-998

KyCL,BarreP,LorieuxM,TrouslotP,AkaVouS,LouarnJ,CharrierA,H amon S,Noirot M(2000) InterspeciWcgeneticlinkagemap,segregationdistortionandge neticconversionincoVee(Coffea sp.).TheorApplGenet101:669-676

LaRotaM,KantetyRV,YuJK,SorrellsME(2005)Nonrandomdistrib utionandfrequenciesofgenomicandESTderivedmicrosatellitemarkersinrice, wheat,andbarley.BMCG e-nomics6:23

LashermesP,CombesMC,PrakashNS,TrouslotP,LorieuxM,Charri erA(2001)GeneticlinkagemapofCoffea canephora:eVectofsegregationdistortionandanalysisofrecom binationrateinmaleandfemalemeioses.Genome44:589-596
LashermesP,CombesMC,TrouslotP,CharrierA(1997)Phylogeneticrelationshipsofco Vee-treespecies(Coffea L.)asinferredfromITSsequencesofnuclearribosomalDNA.TheorAppl Genet94:947-955

LemP,LallemandJ(2003)GrassconsensusSTS markers:aneYcientapproachfordetectingpolymorphisminLolium.TheorAp plGenet107:1113-1122

LiewlaksaneeyanawinC,RitlandCE,ElKassabyYA,RitlandK(2004)Single-copy,speciestransferablemicrosatellitemarkersdevelopedfromloblollypineESTs. TheorApplGenet 109:361 $-369$

LinC,MuellerLA,CarthyJM,CrouzillatD,PetiardV, TanksleySD( 2005)CoVeeandtomatosharecommongenerepertoiresasrevealedbydeepsequencingofseedandcherrytranscripts. TheorApplGenet112:114-130

LiuK,MuseSV(2005)PowerMarker:anintegratedanalysisenvironmentf orgeneticmarkeranalysis.Bioinformatics 21:2128-2129

MetzgarD,BytofJ,WillsC(2000)Selectionagainstframeshiftmutatio nslimitsmicrosatelliteexpansionincodingDNA.GenomeRes10: $72-80$

MoncadaP,McCouchS(2004)Simplesequencerepeatdiversityindipl oidandtetraploidCoffea species.Genome47:501-

509MorganteM,HanafeyM,PowellW(2002)Microsatellitesarepref erentiallyassociated withnonrepetitive DNAinplant genomes.NatGenet30:194-200

N'DiayeA,NoirotM,HamonS,PoncetV(2006)Geneticbasisofspecie sdiVerentiationbetweenCoffea libericaHiernand C.canephoraPierre:analysisofaninterspeciWccross.GenetResou rCropEvol(inpress)

N'DiayeA,PoncetV,LouarnJ,HamonS,NoirotM(2005)GeneticdiVerentiationbetweenCoffea libericavar.libericaand C.libericavarDewevreiandcomparisonwithC.canephora.PlantS ystEvol253:95-104

5)AnSSR-andAFLPbasedgeneticlinkagemapoftallfescue(FestucaarundinaceaSchreb.).Th 110:323-336 eorApplGenet

ParkYH,AlabadyMS,UlloaM,SicklerB,WilkinsTA,YuJ,StellyDM, KohelRJ,el-ShihyOM,CantrellRG(2005)Ge-

neticmappingofnewcottonWberlociusingEST-derivedmicrosatellitesinaninterspeciWcrecombinantinbredlinecottonpo pulation.MolGenetGenomics274:428-441

PintoLR,Oliveira

KM,UlianEC, GarciaAA,deSouzaAP(2004)Surveyinthesugarcaneexpres sedsequencetagdatabase(SUCEST)forsimplesequencerepeats.Genome47:795804

PoncetV,HamonP,MinierJ,CarascoLacombeC,HamonS,NoirotM(2004)SSRcrossampliWcationandvariationwith-incoVeetrees(Coffea spp.).Genome47:1071-1081

RalloP,TenzerI,GesslerC,BaldoniL,DoradoG, MartinA(2003)Transferabilityofolivemicrosatellitelociacrosst hegenusOlea. TheorApplGenet107:940-946

RovelliP,MettulioR,AnthonyF,AnzuetoF,LashermesP,GraziosiG(2 000)MicrosatellitesinCoffea arabicaL.In:SeraT,SoccolCR,PandeyA,RoussosS(eds)CoVeebio -technologyandquality. Kluwer,Netherlands, pp123-133

RozenS,SkaletskyHJ(2000)Primer3ontheWWWforgeneralusersan dforbiologist programmers. In:KrawetzS, MisenerS(eds)Bioinformaticsmethodsandprotocols:methodsinm olecularbiology.HumanaPress, Totowa, pp365-386

SahaMC,MianMA,EujaylI,ZwonitzerJC,WangL,MayGD(2004) Tallfescue EST-SSR markers with transferabilityacrossseveralgrassspecies.TheorApplGenet 109:783791SahaMC,MianR,ZwonitzerJC,ChekhovskiyK,HopkinsAA(200 
SambrookJ,FristchEF,ManiatisT(1989)Molecularcloningalaboratorymanual.ColdSpringHarborLaboratoryedn.Col dSpringHarbor

ScottKD,EgglerP,SeatonG,RossettoM,AblettEM,LeeLS,Henr yRJ (2000) Analysis ofSSRsderivedfromgrapeESTs. TheorApplGenet 100:723726

SethyNK,ChoudharyS,ShokeenB,BhatiaS(2006)IdentiWcationofmicrosatellitemarkersfromCicerreticulatum:molecularvariationandphylogeneticanalysis. TheorApplGenet 112:3 47-357

ThielT,MichalekW,VarshneyRK,GranerA(2003)ExploitingE STdatabasesforthedevelopmentandcharacterizationofgene -derivedSSR-

markersinbarley(HordeumvulgareL.).TheorApplGenet 106:4 $11-422$

VarshneyRK,GranerA,SorrellsME(2005a)Genicmicrosatel-lite markersinplants:featuresandapplications.TrendsBiotechnol23:48-55

VarshneyRK,SigmundR,BornerA,KorzunV,SteinN,SorrellsM E,LangridgeP,GranerA(2005b)InterspeciWctransferabilityandcomparativemappingofbarleyEST-SSRmarkersinwheat,ryeandrice.PlantSci168:195-202

VigourouxY,MitchellS, MatsuokaY, HamblinM,KresovichS,SmithJS,JaquethJ,SmithOS,Doebley J(2005)Ananalysisofgeneticdiversityacrossthemaizegenomeusi ngmicrosat-ellites.Genetics169:1617-1630

WuKS,TanksleySD(1993)Abundance,polymorphismandgenetic mappingofmicrosatellitesinrice.MolGenGenet241:225-235

YuJK,LaRotaM,KantetyRV,SorrellsME(2004)ESTderivedSS Rmarkersforcomparativemappinginwheatandrice.MolGenet Genomics271:742-751 